\title{
The cut-off values for non-fasting routine lipid parameters in presumably healthy 9-11-year-old children
}

\author{
Corresponding author: \\ Łukasz Szternel, Department \\ of Laboratory Medicine, \\ Collegium Medicum, \\ Nicolaus Copernicus University, Torun, \\ Sklodowskiej-Curie 9 Str., \\ 85-094 Bydgoszcz, Poland, \\ e-mail: lukaszszternel@wp.pl
}

Medical Research Journal 2020; Volume 5, Number 1, 15-18 10.5603/MRJ.a2020.0002 Copyright @ 2019 Via Medica ISSN 2451-2591

\begin{abstract}
Disorders of lipid metabolism cause accelerated atherosclerosis and increase cardiovascular risk, which is why lipid profile screening, especially at a young age, should be widely applied.

Aim. The aim of the study was to determine the cut-off points for non-fasting lipid parameters in presumably healthy children aged 9-11 years.

Material and methods. The study was performed with the use of blood samples taken in non-fasting state from 289 school children of both sexes (152 girls and 137 boys). Routine lipid profile was assessed: TC, LDL-C, HDL-C, and triglycerides. Laboratory measurements were performed in serum samples using a biochemical autoanalyser.

Results. In this study we determined the 97.5 percentile values for TC, LDL-C, and triglycerides and the 2.5 percentile values for HDL-C. The upper cut-offs for TC, LDL-C, and triglycerides were found to be 239 $\mathrm{mg} / \mathrm{dL}, 163 \mathrm{mg} / \mathrm{dL}$, and $284 \mathrm{mg} / \mathrm{dL}$, respectively, and the lower cut-off for HDL-C was $37 \mathrm{mg} / \mathrm{dL}$.

Conclusions. The upper range of non-fasting total cholesterol was higher by about $30 \mathrm{mg} / \mathrm{dL}$ compared to fasting state for a similar age range; the cut-off points in non-fasting children for LDL-C and TG were also higher. The lower cut-off for HDL-C was similar compared to fasting state for the respective age range. The determination of the non-fasting cut-off values for routine lipid profile in the paediatric population is essential for the proper evaluation of the cardiovascular risk because using the reference values for adults may cause an incorrect interpretation of the laboratory results.

Key words: lipids, children, non-fasting, cholesterol, cut-off points
\end{abstract}

Med Res J 2020; 5 (1): 15-18

\section{Introduction}

Lipid profile screening at younger age allows early identification of children with lipid disorders resulting from both improper nutrition and unhealthy lifestyle, but it also contributes to the detection of cases that are characterised by genetic alterations typical of some conditions such as familial hypercholesterolaemia (FH) [1]. Evaluation of lipid metabolism in the fasting state can be a stressful situation, not only for adults but also for children [2]. A lot of studies that have been conducted over the last years, including both adults and children, have shown that the concentrations of the majority of lipid parameters measured postprandially change only minimally, and what is more, they reflect accurately the lipid metabolism, which is used in, for instance, cardiovascular risk prediction and assess- ment $[3,4]$. The postprandial state prevails throughout the day; thus, the assessment of lipid parameters enables monitoring of the lipid metabolism during the changes connected with the postprandial periods and the assessment of capacity for clearance of fatty acids [3]. It should be also underlined that dyslipidaemia, obesity, and other cardiovascular risk factors that appear in the youth period pose a serious risk to health in adulthood. The recommendations concerning the evaluation of lipid metabolism in children advise lipid screening among all children aged $9-11$ years. It is indicated that the tests that assess total cholesterol concentration (TC) as well as HDL cholesterol do not require a fasting state $[5,6]$. According to the recommendations of the American Academy of Paediatrics (AAP) from 2011, it is advisable to perform a screening of lipid parameters in children. Conducting such tests 
when children are still in pre-pubertal age is justified for a number of reasons.

Despite the existing recommendations concerning the first assessment of lipid metabolism and a lot of studies indicating only minor differences related to the influence of food intake on the lipid profile parameters, there are no data discussing the proper cut-off values for the lipid profile parameters measured in children in a non-fasting state. The European Atherosclerosis Society (EAS) and the European Federation of Clinical Chemistry and Laboratory Medicine (EFLM) presented in 2016 data concerning the cut-off values for the lipid parameters in a non-fasting state for the adult population only [2]. Thus, there is a gap regarding the paediatric population and resulting concern as to whether the lipid profile analysis in children in a non-fasting state should refer to the reference values established on the basis of a fasting state. This should be regarded as a potential threat for the proper interpretation of lipid profile screening results.

The aim of this study was to establish the cut-off values for the non-fasting lipid profile parameters in presumably healthy children aged 9-11 years. It was done for the routine lipid profile parameters; namely, total cholesterol, HDL cholesterol, LDL cholesterol, and triglycerides.

\section{Subjects and materials}

The study group consisted of 289 presumably healthy children, including 152 girls and 137 boys. An analysis of lipid metabolism was carried out in accordance with the recommendations of the National Heart, Lung, and Blood Institute, the American Paediatric Academy, and the Polish Paediatric Society, recommending the first screening assessment of the parameters of the lipid profile in all children without additional risk in the age group of 9-11 years [7]. The tested material was venous blood collected in non-fasting state. In the studied group of children, the following parameters of the lipid profile were assessed: TC, HDL-C, LDL-C, and TG.
This study was approved by the Bioethics Committee of the Collegium Medicum in Bydgoszcz, Nicolaus Copernicus University in Toruń.

\section{Methods}

The lipid parameters were analysed in serum using the ABX Pentra 400 biochemical platform. The concentration of LDL-C was measured using the direct method. All analysese were made in the Department of Laboratory Medicine, Collegium Medicum of the Nicolaus Copernicus University in Toruń, in accordance with the principles of good laboratory practice.

\section{Statistical analysis}

The reference values were analysed with the MedCalc program (MedCalc Software bvba, Ostend, Belgium; https://www.medcalc.org; 2016) following the CLSI guidelines for the non-parametric percentile method. A 95\% bilateral reference range was determined with a lower limit of the 2.5 percentile and an upper limit of the 97.5 percentile and $90 \%$ confidence intervals for the determined values. Normal distribution charts were made in Microsoft Excel.

\section{Results}

Table 1 presents selected elements of descriptive statistics for parameters of the routine lipid profile of the children included in the study, while Table 2 presents the values of average concentrations of parameters of the lipid profile depending on the BMI centile. The analysed $\mathrm{BMI}$ value ranged from 11.3 to $32.9 \mathrm{~kg} / \mathrm{m}^{2}$. Children with a BMI over the $90^{\text {th }}$ percentile constituted $16.6 \%$ of the study group, which could have resulted in higher cut-off values for total cholesterol, triglycerides, and LDL-C cholesterol. Statistical analysis showed a Gaussian distribution, which indicates that most of

Table 1. Selected elements of descriptive statistics for parameters of the lipid profile

\begin{tabular}{lcccccc}
\hline $\begin{array}{l}\text { Lipid } \\
\text { parameters } \\
\text { (units) }\end{array}$ & $\begin{array}{c}\text { Standard } \\
\text { Mean }\end{array}$ & $\begin{array}{c}\text { Standard } \\
\text { deviation }\end{array}$ & Median & $\begin{array}{c}\text { Maximum } \\
\text { concentration }\end{array}$ & $\begin{array}{c}\text { Minimum } \\
\text { concentration }\end{array}$ & $\begin{array}{c}\text { Skewness } \\
\text { factor }\end{array}$ \\
\hline TC $(\mathrm{mg} / \mathrm{dL})$ & 171.4 & 31.0 & 170.5 & 295.0 & 94.0 & 0.49 \\
TG $(\mathrm{mg} / \mathrm{dL})$ & 107.8 & 58.5 & 91.7 & 353.7 & 29.7 & 1.71 \\
HDL-C $(\mathrm{mg} / \mathrm{dL})$ & 58.2 & 13.2 & 57.1 & 130.9 & 27.6 & 0.98 \\
LDL-C $(\mathrm{mg} / \mathrm{dL})$ & 97 & 27.5 & 95.1 & 218.0 & 26.3 & 0.85 \\
\hline
\end{tabular}

TC — total cholesterol; TG — triglycerides; HDL-C — high-density lipoprotein cholesterol; LDL-C — low-density lipoprotein cholesterol 
Table 2. Values of average concentrations of parameters of the lipid profile depending on the BMI centile

\begin{tabular}{lccccccc}
\hline BMI centile & \multicolumn{7}{c}{ Lipid parameters (units) } \\
\cline { 2 - 8 } & $\begin{array}{c}\text { TC } \\
{[\mathbf{m g} / \mathbf{d l}]}\end{array}$ & $\begin{array}{c}\text { TG } \\
{[\mathbf{m g} / \mathbf{d l}]}\end{array}$ & $\begin{array}{c}\text { HDL-C } \\
{[\mathbf{m g} / \mathbf{d l}]}\end{array}$ & $\begin{array}{c}\text { LDL-C } \\
\text { [mg/dl] }\end{array}$ & $\begin{array}{c}\text { Lp/a) } \\
\text { [mg/dl] }\end{array}$ & $\begin{array}{c}\text { apoB } \\
\text { [g/l] }\end{array}$ & $\begin{array}{c}\text { apoA1 } \\
\text { [g/l] }\end{array}$ \\
\cline { 2 - 8 } BMI centile <10 & 169.3 & 91.9 & 64.1 & 89.9 & 14.3 & 0.77 & 1.26 \\
BMI centile 10-90 & 171.7 & 98.6 & 59.3 & 96.7 & 17.9 & 0.81 & 1.24 \\
BMI centile > 90 & 172.0 & 160.9 & 48.8 & 104.1 & 20.2 & 0.87 & 1.14 \\
p & 0.97 & $<0.001$ & $<0.001$ & 0.09 & 0.14 & 0.02 & 0.001 \\
\hline
\end{tabular}

$\mathrm{BMI}$ — body mass index; TC — total cholesterol; TG — triglycerides; HDL-C — high-density lipoprotein cholesterol; LDL-C — low-density lipoprotein cholesterol; $\mathrm{p}$ - significance of differences between BMI centile category

the results from the study group are smaller than the determined mean. Below are presented the results of analyses determining the cut-off points for selected lipid parameters in the population of non-fasting children aged 9-11 years. The upper cut-off points for total cholesterol, triglycerides, and LDL-C in non-fasting children aged 9-11 years were $238.7 \mathrm{mg}$ /dL, 90\% Cl, (230.7-262.9 mg /dL); 283.9 mg/dL, 90\% Cl $(245.4-335.2 \mathrm{mg} / \mathrm{dL})$; and $162.9 \mathrm{mg} / \mathrm{dL}, 90 \% \mathrm{Cl}$ (147.9-186 mg/dL), respectively. The lower cut-off point for HDL-C in non-fasting children aged 9-11 years was $36.5 \mathrm{mg} / \mathrm{dL}, 90 \% \mathrm{Cl}(31.3-38.6 \mathrm{mg} / \mathrm{dL})$.

\section{Discussion}

The obesity epidemic, as well as the growing risk of type 2 diabetes and arterial hypertension among the paediatric population, emphasise the importance of laboratory analysis of lipid metabolism, which is a vital part of preventive medicine. Children with excessive body mass belong to a higher risk category when it comes to developing atherosclerosis, and the evaluation of lipid metabolism should be a standard tool used to identify lipid disorders [7]. In the following study, it was determined that the value for: TC $(239 \mathrm{mg} / \mathrm{dL}), \mathrm{TG}$ (284 mg/dL), and LDL-C (163 mg/dL) is the 97.5 percentile, and the value for HDL-C: $(37 \mathrm{mg} / \mathrm{dL}$ ) is the 2.5 percentile. It should be highlighted that we used paired samples to estimate lipid differences between fasting and non-fasting state, which is an unquestionable advantage of this study.

The results of the study revealed that the cut-off values among children in a non-fasting state, for the total cholesterol, are higher by about $30 \mathrm{mg} / \mathrm{dL}$ (upper cut-off value in fasting state was $200 \mathrm{mg} / \mathrm{dl}$ ), while the TG concentration is 2.5-fold higher (upper cut-off value in fasting state for age 0-9 years was $100 \mathrm{mg} / \mathrm{dl}$ and for $10-19$ years was $130 \mathrm{mg} / \mathrm{dl}$ ) in comparison to determinations performed in a fasting state for similar age range groups [8]. What is more, the higher cut-off value was also different for the LDL-C concentration in comparison to that measured in a non-fasting state in the adult population. The HDL-C concentration was only slightly lower when compared to the cut-off values suggested by EAS/EFCCLM [2]. The research related to the evaluation of the lipid parameters in a non-fasting state, which was conducted in the adult population, showed that the concentrations of lipids, lipoproteins, and apolipoproteins changed only in a minor way in response to food intake for people in the general population. The evaluation of lipid parameters measured in a non-fasting state is a good indicator of the risk of cardiovascular events; what is more, there have been no unambiguous studies that point to a better diagnostic use of lipid screening conducted in a fasting state in comparison to the analysis performed in individuals in a non-fasting state. Evaluating the lipid profile parameters in children with the use of the reference values established for adults can pose a risk of incorrect interpretation of laboratory results. The study conducted by the National Health and Nutrition Examination Survey (NHANES) provided some useful data related to the subject of distribution and trends in the concentrations of lipids and lipoproteins during childhood and adolescence [5]. The average concentration of cholesterol in children aged 9-11 years was established at the level of $171 \mathrm{mg} / \mathrm{dl}$, and a lower concentration was observed in adolescence. It is of vital importance for the age range of lipid profile screening and the used cut-off values because the concentrations of lipid parameters depend on age and puberty period [9]. The concentration of HDL cholesterol was similar in our study and in the study of Nordestgaard et al. [2]. The values obtained in the following study are comparable or slightly higher in comparison to the results presented in the CALIPER project, in which the paediatric reference ranges were set for a number of diagnostic parameters, including the parameters of the lipid profile. Additionally, the CALIPER project was conducted among a much larger study group than the following study. The differences in lipid concentration levels may result from the difference in age ranges and the strictly standardised meal used in the CALIPER study [10]. Boyd et al. demonstrated 
that obesity and hypertension occurring in childhood have a negative impact on the lipid metabolism. They also drew attention to the necessity of performing lipid profile screening not only in obese children who suffer from hypertension or diabetes, but in all children with excessive body mass [11].

To summarise, the determined cut-off values for TC, TG, and LDL-C concentrations in a non-fasting state are higher, while the determined cut-off values for HDL-C are lower than the cut-off values for the concentrations of these parameters measured in a fasting state. It has been presented that the concentrations of the lipid profile parameters measured in children aged 9-11 years depend on age and body mass. Abnormal body mass in childhood and the related disorders of the lipid metabolism may increase significantly the risk of cardiovascular diseases in adulthood, and measurement of lipid profile parameters is one of the most basic tests used in cardiovascular risk assessment. In light of numerous cardiovascular events and the resulting high mortality rate, there is an urgent need for common lipid profile parameter screening beginning at paediatric age.

\section{Conclusion}

The non-fasting cut-off value of total cholesterol concentration was higher and the cut-offs for LDL-C and TG were significantly higher in comparison to the concentrations in a fasting state for the corresponding age range. The determination of the specific lipid profile cut-off values for the paediatric population is essential for the proper evaluation of the cardiovascular risk because using the reference values for adults may cause incorrect interpretation of the laboratory results.

Abbreviations: TC - total cholesterol, HDL-C - high-density lipoprotein cholesterol, LDL - lowdensity lipoprotein cholesterol, sd-LDL-C - small, dense, low-density lipoprotein cholesterol, apoAI - apolipoprotein AI, apoB - apolipoprotein B, $\mathrm{Lp}(\mathrm{a})$ - lipoprotein (a), BMI — body mass index, $\mathrm{FH}$ - familial hypercholesterolaemia, CM UMK - Collegium Medicum University of Nicolaus Copernicus, CLSI - Clinical and Laboratory
Standards Institute, AAP - American Academy of Paediatrics, EAS/EFCCLM - European Atherosclerosis Society/European Federation of Clinical Chemistry and Laboratory Medicine, NHANES - National Health and Nutrition Examination Survey

\section{References}

1. Expert Panel on Integrated Guidelines for Cardiovascular Health and Risk Reduction in Children and Adolescents, National Heart, Lung, and Blood Institute. Expert panel on integrated guidelines for cardiovascular health and risk reduction in children and adolescents: summary report. Pediatrics. 2011; 128 Suppl 5: S213-S256, doi: 10.1542/peds.20092107C, indexed in Pubmed: 22084329.

2. Nordestgaard B, Langsted A, Mora S, et al. Fasting is not routinely required for determination of a lipid profile: clinical and laboratory implications including flagging at desirable concentration cut-points-a joint consensus statement from the European Atherosclerosis Society and European Federation of Clinical Chemistry and Laboratory Medicine. European Heart Journal. 2016; 37(25): 1944-1958, doi: 10.1093/eurheartj/ehw152.

3. Steiner MJ, Skinner AC, Perrin EM. Fasting might not be necessary before lipid screening: a nationally representative cross-sectional study. Pediatrics. 2011; 128(3): 463-470, doi: 10.1542/peds.2011-0844, indexed in Pubmed: 21807697.

4. Langsted A, Freiberg JJ, Nordestgaard BG. Fasting and nonfasting lipid levels: influence of normal food intake on lipids, lipoproteins, apolipoproteins, and cardiovascular risk prediction. Circulation. 2008; 118(20): 2047-2056, doi: 10.1161/CIRCULATIONAHA.108.804146, indexed in Pubmed: 18955664

5. Expert Panel on Integrated Guidelines for Cardiovascular Health and Risk Reduction in Children and Adolescents: Summary Report. PsycEXTRA Dataset. 2012, doi: 10.1037/e531652012-001.

6. Brothers JA, Daniels SR. Special PatientPopulations: Children and Adolescents Ballantyne Christie M. Clinical Lipidology: A Companion to Braunwald's Heart Disease 2nd edition. Elsevier Sanders, Philadelphia. ; 2015: 427-429.

7. Daniels SR, Greer FR. Committee on Nutrition. Lipid screening and cardiovascular health in childhood. Pediatrics. 2008; 122(1): 198-208, doi: 10.1542/peds.2008-1349, indexed in Pubmed: 18596007.

8. Szternel L, Krintus M, Bergmann K, et al. Non-fasting lipid profile determination in presumably healthy children: Impact on the assessment of lipid abnormalities. PLoS One. 2018; 13(6): e0198433, doi: 10.1371/journal.pone.0198433, indexed in Pubmed: 29927973.

9. Hickman TB, Briefel RR, Carroll MD, et al. Distributions and trends of serum lipid levels among United States children and adolescents ages 4-19 years: data from the Third National Health and Nutrition Examination Survey. Prev Med. 1998; 27(6): 879-890, doi: 10.1006/pmed.1998.0376, indexed in Pubmed: 9922071.

10. Adeli K, Higgins V, Trajcevski K, et al. The Canadian laboratory initiative on pediatric reference intervals: A CALIPER white paper. Crit Rev Clin Lab Sci. 2017; 54(6): 358-413, doi: 10.1080/10408363.2017.1379945, indexed in Pubmed: 29017389.

11. Boyd GS, Koenigsberg J, Falkner B, et al. Effect of obesity and high blood pressure on plasma lipid levels in children and adolescents. Pediatrics. 2005; 116(2): 442-446, doi: 10.1542/peds.2004-1877, indexed in Pubmed: 16061601. 